the junior doctors in the prescription of MRAs however $(\mathrm{p} \leq 0.05)$. Self-reported competency in initiating and up-titrating $\mathrm{HF}$ medications is reported in table 2. Of participants who felt comfortable with initiation and up-titration of beta blockers ( $\mathrm{N}=89)$, only $26 \%(\mathrm{~N}=27)$ correctly identified an optimal target heart rate of less than 70 beats per minute. Twenty-four percent of respondents $(\mathrm{N}=28)$ were unaware of a specialist HF service that catered to their institution, and how to refer to it, but $97 \%(\mathrm{~N}=113)$ felt that their practice would benefit from further education on HF pharmacotherapy.

Conclusion The high prevalence of HF in Ireland and costs associated with admission for decompensation necessitates a sound knowledge of its management amongst generalists. Results of this survey suggest a need, and indeed a demand, for further education and support surrounding pharmacotherapy of stable heart failure.

\section{APICAL HYPERTROPHIC CARDIOMYOPATHY: THE VARIANT LESS KNOWN}

H Cronin, C Galvin, M Gallagher, J Galvin, C McGorrian. Mater Misericordiae University Hospital Dublin. Ireland

\subsection{6/heartjnl-2021-ICS.31}

Introduction Hypertrophic cardiomyopathy (HCM) is a heterogenous disease. Apical HCM is a comparatively rare subtype. Apical HCM tends to be sporadic with different clinical features and lower frequency of detected gene mutations [figure 1]. We evaluated the spectrum of apical HCM-associated gene mutations, genotype-phenotype correlations, and clinical outcomes of patients in our cohort.

Methods 30 patients (29 probands) with apical HCM were retrospectively identified at a specialist cardiomyopathy clinic. Records were reviewed and variables were recorded including: demographics, clinical characteristics, family history, presenting symptoms, device therapy, arrhythmia burden, and other comorbidities. ECG, cardiac imaging and genetic test results were analyzed.

Results The group consisted of 21/30 (70\%) men; mean age 57 years. 3 probands $(10.3 \%)$ in the study had a family history of HCM. Another 3 probands (10.3\%) had a family history of sudden cardiac death in a first degree relative. Women were older than men at initial visit, 57 years versus 49 years. Of the 26 patients who had an ECG available for analysis, all were abnormal by established criteria. Female patients were more likely to have a family history of HCM $(22 \%$ versus $4.7 \%$ of males). They have a higher burden of symptoms and higher rates of mid-cavity obstruction compared to males

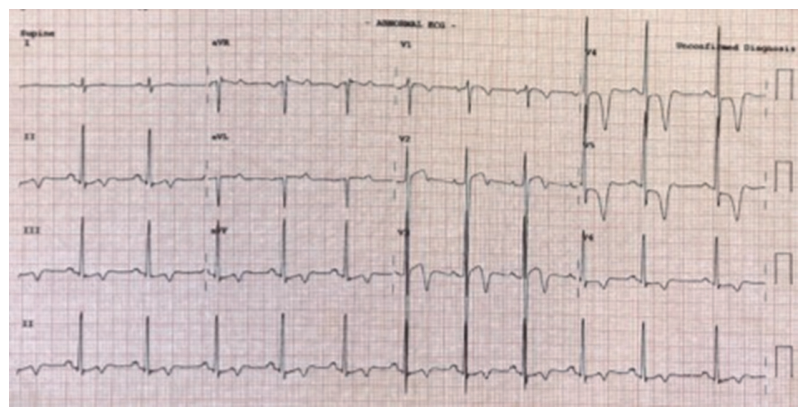

Abstract 31 Figure 1 Apical HCM ECG
Abstract 31 Table 1 Genotypes of apical HCM

\begin{tabular}{llllll}
\hline AAD & Sex & Presentation & FHx & SCD & Mutation \\
\hline 63 & F & Palpitations & No & No & MYBPC3 p.Arg502Gln \\
68 & F & Palpitations & No & No & MYH7 p.Arg1079Gln \\
66 & F & Palpitations & No & No & TNNI3 p. Arg162Gln \\
61 & F & Palpitations & Apical & No & MYBPC3 p.Lys814del \\
21 & F & Syncope & Septal & No & TNNI3 p.Arg 141Gln \\
16 & F & Syncope & LVNC & Yes & ACTC1 p.Glu101 Lys \\
41 & M & Family & Apical & No & MYBPC3 \\
& & screening & & & C.2441_2443delAga \\
59 & M & Chest pain & No & No & PTPN11 p.Thr468Met \\
47 & M & Chest pain & No & No & SCL22A5 p. Arg83Le
\end{tabular}

AAD: age at diagnosis, FHx : family history of apical or classical HCM or other cardiomyopathy, SCD in a first degree relative from HCM

(88\% versus $33 \%)$. Genetic analysis was performed on 24/30 patients $(80 \%)$. The results are summarized in table $1.75 \%$ of female probands had a pathogenic variant in a sarcomere gene. No male proband had a sarcomere mutation. One male relative of a female proband inherited the familial MYBPC3 mutation. Conclusion The two most commonly affected genes in our cohort were MYBPC3 (3 patients) and TNNI3 (2 patients), with mutations also found in MYH7, ACTC1, PTPN11 and SCL22A5 [table 1]. De-novo mutations in ACTC1, specifically the p.Glu101Lys variant, have been shown to consistently produce an apical HCM phenotype. Apical HCM is a global HCM variant with phenotypic expression that is modulated by environmental and genetic factors. There was a striking variation in the frequency of causative gene variant identification between probands by sex. This may relate to the interplay of genetic, epigenetic and polygenic factors. This finding warrants further investigation, and may inform future family screening and genetic testing strategies.

\section{PREVALENCE AND CHARACTERISTICS OF ABNORMALITIES ON CARDIAC MRI IN PRIMARY CARE FOLLOWING RECOVERY FROM ACUTE SARS-COV-2 INFECTION AND CORRELATION WITH MARKERS OF IMMUNITY AND COAGULATION: PRIMARY RESULTS OF THE SETANTA STUDY}

\begin{abstract}
${ }^{1,2} \mathrm{R}$ Colleran, ${ }^{1} \mathrm{R}$ Byrne, ${ }^{3} \mathrm{~A}$ Cradock, ${ }^{4} \mathrm{D}$ O'Ciardha, ${ }^{5} \mathrm{~S} \mathrm{Mc}$ Keogh, ${ }^{1,6} \mathrm{H}$ Wilson, ${ }^{7} \mathrm{~A}$ Mansoor, ${ }^{1} \mathrm{~A}$ Carswell, ${ }^{1} \mathrm{C}$ Farrelly, ${ }^{8} \mathrm{M}$ Hannan, ${ }^{9} \mathrm{M}$ Fitzgibbon, ${ }^{10} \mathrm{~J}$ O'Donnell, ${ }^{1,2} \mathrm{H}$ Rai, ${ }^{1,11} \mathrm{RA}$ Byrne. ${ }^{1}$ Cardiovascular Research Institute Dublin, Mater Private Network, Ireland; ${ }^{2}$ School of Medicine, RCSI University of Medicine and Health Sciences, Ireland; ${ }^{3}$ Department of Medicine, University College Dublin, Ireland: ${ }^{4}$ Institute of Population Health, Trinity College Dublin, Ireland; ${ }^{5}$ Solas Medical Centre, Dublin, Ireland; ${ }^{6}$ School of Nursing \& Midwifery, RCSI University of Medicine and Health Sciences, Ireland; ' ${ }^{7}$ Mater Private Network, Ireland; ${ }^{8}$ Department of Pathology, Mater Private Network, Ireland; ${ }^{9}$ Mater Private Network, Ireland and School of Medicine, University College Dublin, Ireland; ${ }^{10}$ Irish Centre for Vascular Biology, RCSI University of Medicine and Health Sciences, Ireland; ${ }^{11}$ School of Pharmacy and Biomolecular Sciences, RCSI University of Medicine and Health Sciences, Ireland
\end{abstract}

\subsection{6/heartjnl-2021-ICS.32}

Introduction Tissue characterization using cardiac magnetic resonance (CMR) imaging has the potential to delineate early signs of inflammatory cardiomyopathy. Recent CMR studies in patients recovered from SARS-CoV-2 patients suggested a high prevalence of abnormal findings on CMR, including lower left ventricular ejection fraction, higher left ventricle volumes, and raised native T1 and T2 signal values. Concerns persist, 